\title{
Two degree-of-freedom parallel mechanisms for high bandwidth vibration suppression and tracking
}

\author{
Dustin J. Carruthers $^{a}$ and John F. O'Brien ${ }^{a}$ \\ ${ }^{a}$ Department of Electrical Engineering, University of Wyoming, Department 3295, $1000 \mathrm{E}$ \\ University Ave, Laramie, WY, 82071, USA
}

\begin{abstract}
Limited degree-of-freedom (DOF) parallel kinematic mechanisms (PKM) are capable of high bandwidth tracking and disturbance rejection with smaller actuators than are required for serial machines. A high performance Nyquist-stable (NS) controller with nonlinear dynamic compensation (NDC) applied to a new PKM is presented. The Popov criterion is used to show absolute stability (AS) of the closed loop system and experimental evidence of closed loop performance is provided. This novel approach to PKM control provides $38 \mathrm{~dB}$ of disturbance rejection below $10 \mathrm{~Hz}$, an order of magnitude more that what is achieved by implementing an absolutely stable fixed gain (ASFG) controller.
\end{abstract}

Keywords: parallel kinematic mechanism, vibration suppression, disturbance rejection, nonlinear dynamic compensation, Nyquist stable, absolute stability, Popov criterion

\section{INTRODUCTION}

Gimbals are frequently used in two-DOF pointing applications. ${ }^{1,2}$ As these are serial mechanisms, the base actuator must provide sufficient torque to move the second motor as well as the end-effector. A parallel machine shares the load amongst the actuators in a closed kinematic chain, allowing for smaller actuators and providing higher bandwidth. Parallel mechanisms have been developed for high performance camera orientation and vibration isolation. Some have gone as far as mimicking the mechanics of the human eye through tendon driven parallel architectures. Proportional-integral compensation is used to control tendon tension for the four extraocular muscles of the robotic MAC-EYE. ${ }^{3}$ The Agile Eye is a 3 -RRR spherical parallel wrist in which all pairs of adjacent joint axes are orthogonal. ${ }^{4}$ Bonev et al. reports that the mechanism has unlimited orientation workspace and has self-motion singularities confined to six singularity curves. ${ }^{5}$ A 3-PUP mechanism delivers a translation and a rotation task DOF, with an additional parasitic translation. ${ }^{6}$ Vertechy and Parenti-Castelli present an interesting analysis of over-constrained parallel mechanisms obtained from architecturally singular US mechanisms by adding legs consisting of universal joints, active prismatic joints and spherical joints. ${ }^{7}$ A new class of limited DOF PKM designs using a combination of fast prismatic and revolute actuators for vibration suppression applications is presented by Nelson. ${ }^{8}$

Limited DOF PKMs are capable of fast, accurate motion, making them well suited for closed loop tracking/vibration suppression applications. Lower bound stiffness control is used on a 2 DOF 3 - $\underline{R} R R$ mechanism as reported by Kock and Schumacher. ${ }^{9}$ A 3 UPS-PU mechanism has been used for two-DOF vibration suppression. ${ }^{10}$ The purely passive PU center strut restricts end effector movement to one linear translation and two orthogonal rotations. A two axis controller provided $27 \mathrm{~dB}$ of disturbance rejection for the rotational DOFs over the frequency interval 1-10 Hz.

Nonlinear control increases the available feedback for a given bandwidth restriction. ${ }^{11}$ O'Brien applied a Nyquist-stable controller with nonlinear dynamic compensation to the problem of rudder roll stabilization (RRS) of ships. ${ }^{12,13}$ The approach provides substantial roll reduction improvement compared to linear controllers. A Nyquist-stable pointing control system with nonlinear dynamic compensation applied to a limited DOF PKM is described in this paper. Despite a $0 \mathrm{~dB}$ crossover restriction less than $100 \mathrm{~Hz}$, the controller provides nearly 40 $\mathrm{dB}$ of feedback over a $10 \mathrm{~Hz}$ functional bandwidth and satisfies the conditions of absolute stability in actuator saturation.

Further author information: (Send correspondence to D.J.C.)

D.J.C.: E-mail: dustinc@uwyo.edu, J.F.O.: E-mail: obrienj@uwyo.edu, Telephone: 1 (307) 766-2162

Active and Passive Smart Structures and Integrated Systems 2011, edited by Mehrdad N. Ghasemi-Nejhad, Proc. of SPIE Vol. 7977, $797708 \cdot$ ? C 2011 SPIE · CCC code: 0277-786X/11/\$18 · doi: 10.1117/12.880064 


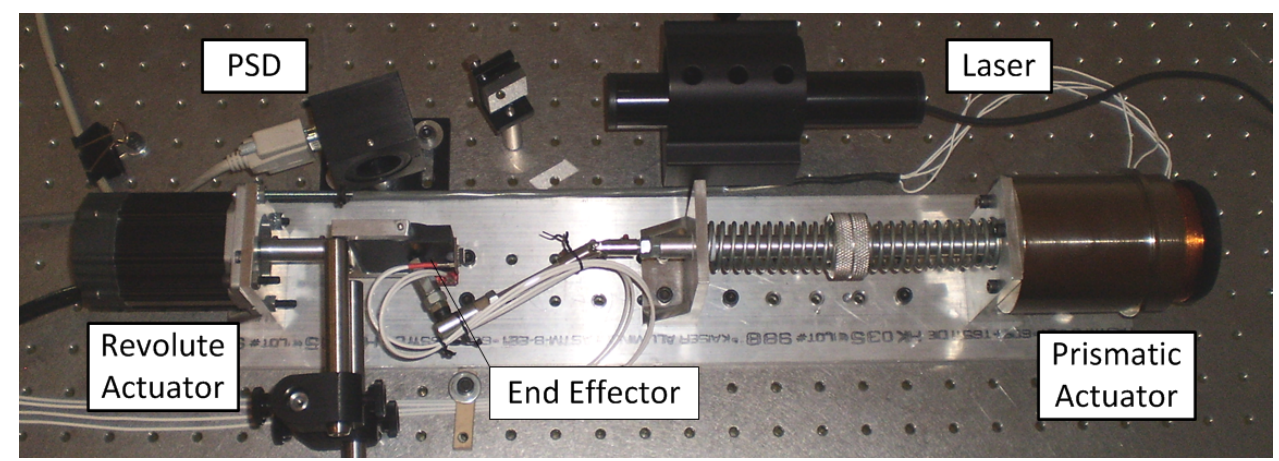

Figure 1. $\underline{P} U S-\underline{R} R$ Parallel Robot

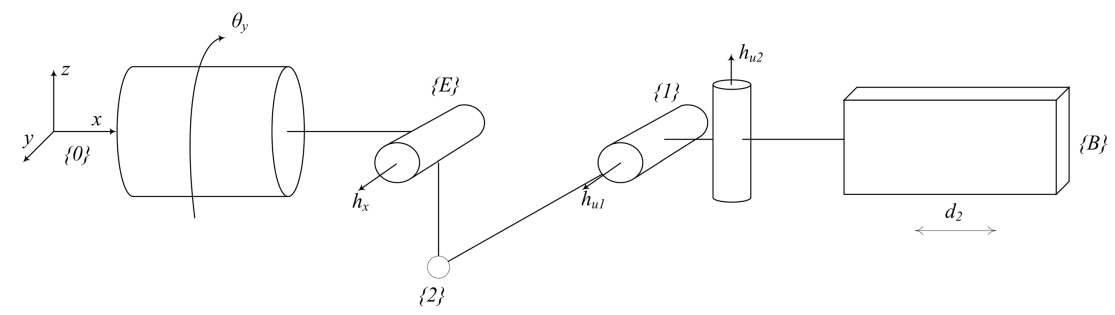

Figure 2. $\underline{P} U S-\underline{R} R$ Parallel Robot Schematic

\section{2. $\underline{P} U S-\underline{R} R$ MECHANISM}

\subsection{Architecture}

The plant is a $\underline{P} U S-\underline{R} R$ parallel robot shown in Figures 1 and $2 .^{8}$ One kinematic path consists of an active prismatic actuator, universal joint and sphere joint $(\underline{P} U S)$, while the other is an active revolute joint in series with a pin joint $(\underline{R} R)$.

\subsection{Actuation}

The prismatic actuator is powered by a linear voice coil manufactured by BEI Kimco Magnetics Division, model number LA28-43-000A. It is capable of around $300 \mathrm{~N}$ of force and $3 \mathrm{~cm}$ of displacement. The coil is mechanically in series with helical springs that provide a restoring force to keep the end effector centered when unpowered. The coil is driven by an AE Techron LV608 Linear Power Amplifier operating in a voltage controlled voltage source mode with a gain of $30 \mathrm{~V} / \mathrm{V}$. The revolute actuator is a standard NEMA 23 sized electric motor by Techic driven by an SSt-Eclipse digital controller running in torque control mode. This DOF is not used for the control experiments described in this paper.

\subsection{Sensing}

Attached to the end-effector are two microelectromechanical system (MEMS) gyros used to measure the angular rate about the two DOFs. These Analog Devices ADXRS610 $300^{\circ}$ single axis gyroscope sensors have an operational bandwidth of $360 \mathrm{~Hz}$. They work by measuring the deflection experienced by a proof mass resonating at $14 \mathrm{kHz}$ caused by the Coriolis effect. This deflection measurement is amplified and demodulated to provide a voltage output proportional to the angular rate. The quiescent noise power spectrum of the gyro, with a peak at $14 \mathrm{kHz}$, can be seen in Figure 3 . A time delay of around $0.75 \mathrm{~ms} \approx 1 /(1.4 \mathrm{kHz})$ is also observed, and it is suspected that this is introduced by the demodulation. Such nonminimum phase delay limits the attainable bandwidth, and thus are not used for closed loop experimentation.

A Helium Neon (HeNe) laser is used in conjunction with an ON-TRAK Photonics OT-324 Position Sensitive Detector (PSD) to measure the end effector position. The stationary HeNe laser emits a $632.8 \mathrm{~nm}$ beam that is directed by an adjustable steering mirror towards the end effector. A mirror attached to the end effector deflects the beam onto the bare duolateral photodiode sensor of the PSD. The PSD sensing amplifier reports the 


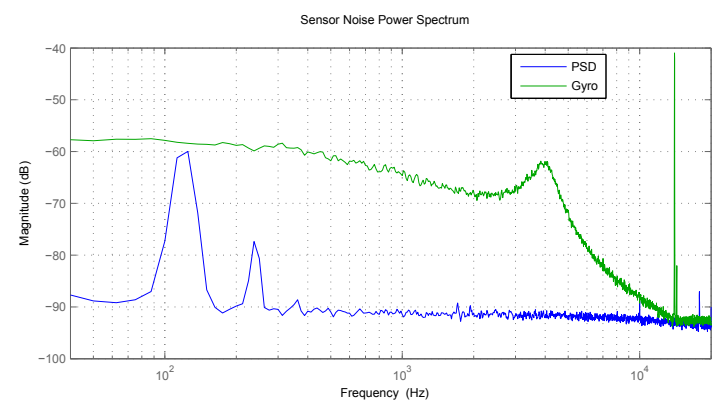

Figure 3. Quiescent Sensor Noise Power Spectrum Densities

position of the laser beam on the two dimensional sensor as two analog voltages within $\pm 10 \mathrm{~V}$. Figure 3 shows the quiescent noise power spectrum of the position sensor along one axis. There are peaks in the position sensors spectra at $120 \mathrm{~Hz}, 240 \mathrm{~Hz}$, and $360 \mathrm{~Hz}$. These harmonics are likely caused by a $120 \mathrm{~Hz}$ voltage ripple on the high voltage DC line powering the laser that results from rectifying the $60 \mathrm{~Hz} \mathrm{AC}$ power. The higher operational bandwidth of nearly $15 \mathrm{kHz}$ and lower noise characteristics make the PSD better suited than the gyro for the control experiments discussed in this paper.

\subsection{System Identification}

The system is stimulated by driving the prismatic actuator with band-limited Gaussian noise for the purpose of system identification. The stimulus and PSD signals are digitized and recorded using an Agilent VXI system. The data capture and processing is performed using the SignalCalc 6.2 software suite from DataPhysics. Stimuli of varying amplitudes and bandwidth are applied and the corresponding coherence and plant transfer functions are calculated. A nominal pole-zero-gain (PZK) model of the plant is developed by the manual placement of poles and zeros to sufficiently match those transfer functions with the highest coherence. This 15th order nominal model $P(s)$ is plotted along with the experimentally acquired data in Figure 4. The gain, zeros, and poles of $P(s)$ are $K=4.4 \cdot 10^{20}, s_{z}=(-5027,-2.482 \pm 496.4 i,-904.8 \pm 2877 i,-108.1 \pm 5402 i)$ and $s_{p}=(-25.13,-24.82 \pm$ $495.8 i,-301.6 \pm 402.1 i,-1319 \pm 2285 i,-184.7 \pm 2632 i,-257.6 \pm 5146 i,-457.4 \pm 6518 i,-326.7 \pm 8162 i)$.

The plant transfer function is low pass as expected. The plant modulus is relatively flat with a value around $48 \mathrm{~dB}$ from $0 \mathrm{~Hz}$ to around $4 \mathrm{~Hz}$, at which point it begins to roll off at approximately $6 \mathrm{~dB} /$ oct. At $80 \mathrm{~Hz}$

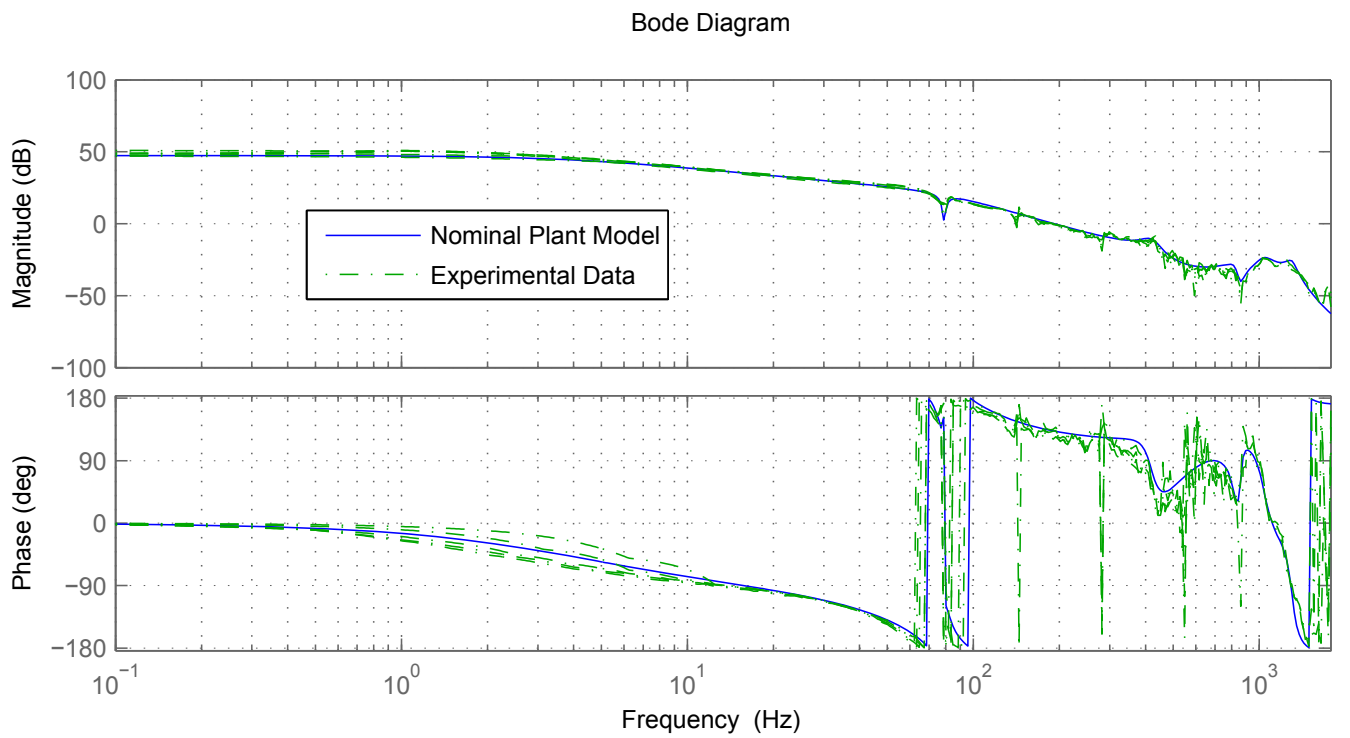

Figure 4. $\underline{P} U S-\underline{R} R$ Plant Frequency Response 


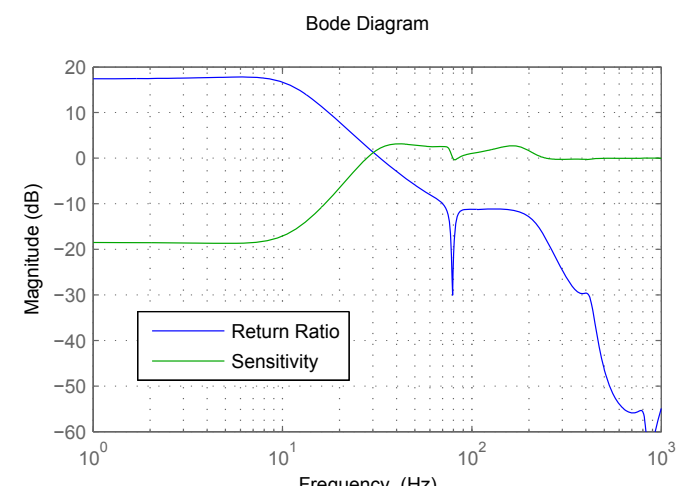

(a) Bode Plot

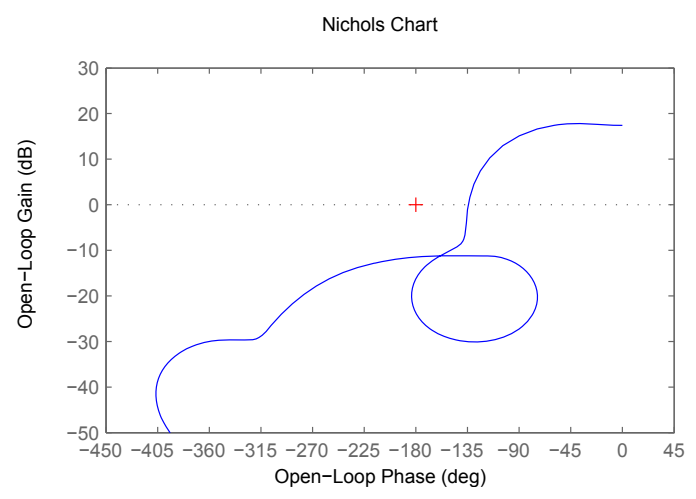

(b) Nichols Plot

Figure 5. ASFG Controller Design

the slope transitions to nearly $18 \mathrm{~dB} /$ oct as there are two conjugate pole pairs and one very lightly damped conjugate zero pair around this frequency. There are interlaced pole then zero pairs around $440 \mathrm{~Hz}$ and $840 \mathrm{~Hz}$. There are consecutive pole pairs at $1 \mathrm{kHz}$ and $1.3 \mathrm{kHz}$, whose addition total phase contribution of $-360^{\circ}$ places a hard upper limit on control bandwidth and thus the amount of available feedback. The phase contributions of the well damped poles and lightly damped poles around $80 \mathrm{~Hz}$ likewise limit control bandwidth as described later in Section 3.1.

\section{CONTROLLER DESIGN AND ANALYSIS}

\subsection{Absolutely Stable Fixed Gain (ASFG) Controller Design}

An absolutely stable fixed gain (ASFG) controller is designed to serve as a baseline for comparison to the nonlinear controller described later. The design goal is to maximize vibration suppression at frequencies below $10 \mathrm{~Hz}$ while remaining absolutely stable. This is accomplished by carefully shaping the compensator $C_{A S F G}(s)$ to obtain the return ratio $T_{A S F G}(s)=C_{A S F G}(s) \cdot P(s)$ seen in Figure 5. Similar to the development of the nominal plant model, the PZK model for $C_{A S F G}$ is designed by manually placing poles and zeros.

A zero at $4 \mathrm{~Hz}$ and a conjugate pair of zeros at $80 \mathrm{~Hz}$ compensate for the corners found in the original plant. A pair of conjugate poles placed at $11 \mathrm{~Hz}$ define the corner of the functional bandwidth around $10 \mathrm{~Hz}$. A pair of zeros at $100 \mathrm{~Hz}$ together with pairs of poles at $160 \mathrm{~Hz}$ and $220 \mathrm{~Hz}$ define the Bode step. ${ }^{11}$ A lead filter with a zero at $60 \mathrm{~Hz}$ and pole at $100 \mathrm{~Hz}$, together with the Bode step, provide sufficient phase advance around the crossover point. Thus a gain margin of at least $10 \mathrm{~dB}$ and a phase margin of nearly $50^{\circ}$ is maintained. This may seem excessive, but it allows us to demonstrate absolute stability (AS) as shown in Section 3.4. The gain, zeros, and poles of $C_{A S F G}(s)$ are $K=196, s_{z}=(-25.13,-377,-301.6 \pm 402.1 i,-502.7 \pm 377 i)$ and $s_{p}=(-628.3,-41.47 \pm 55.29 i,-502.7 \pm 870.6 i,-414.7 \pm 1319 i)$.

A major factor limiting bandwidth is the lightly damped zeros and heavily damped poles around $80 \mathrm{~Hz}$. The phase lag of the poles is more pronounced than the phase lead of the zeros at frequencies below $80 \mathrm{~Hz}$. Thus the phase sharply drops to nearly $-180^{\circ}$ around $80 \mathrm{~Hz}$. There is also uncertainty in the exact frequency and Q factor of this mode, as it varied between different system identification experiments. Thus the mode is gain stabilized in order to retain adequate phase margins. The return ratio crosses $0 \mathrm{~dB}$ around $33 \mathrm{~Hz}$ and the resulting 7 th order controller provides nearly $18 \mathrm{~dB}$ of negative feedback below $10 \mathrm{~Hz}$.

Return ratios $T_{A S F G}$ and sensitivities $S_{A S F G}=1 /\left(1+T_{A S F G}\right)$ are also calculated using the experimentally found plant response data to verify that the final compensator design is acceptable when the plant differs from the nominal model. In all cases, stability is retained and the modulus of positive feedback (where $\left|S_{A S F G}\right|>0$ $\mathrm{dB}$ ) is less than $5 \mathrm{~dB}$. Absolute stability of the ASFG controller is shown in Section 3.4. 


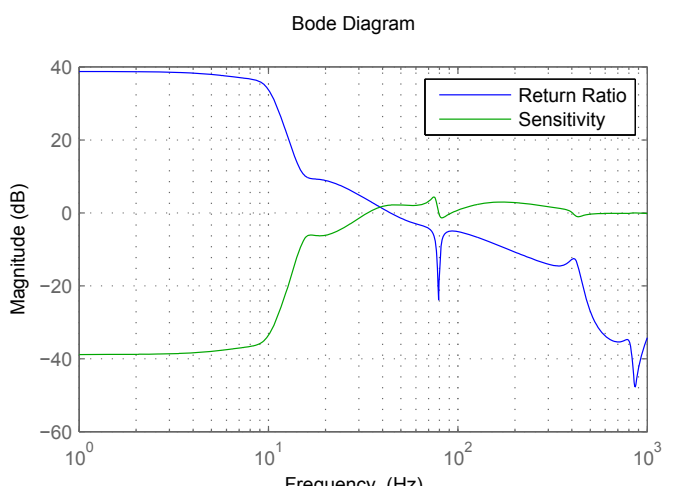

(a) Bode Plot

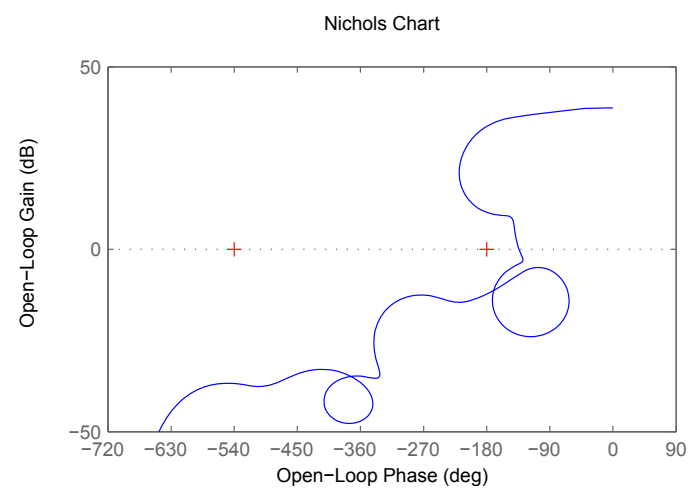

(b) Nichols Plot

Figure 6. NS Controller Design

\subsection{Nyquist Stable (NS) Controller Design}

A high performance Nyquist stable (NS) controller $C_{N S}(s)$ is also designed with the goal of maximizing disturbance rejection at frequencies below $10 \mathrm{~Hz}$. By definition, the return ratio of a Nyquist stable system has a modulus greater than unity and phase less than 180 degrees over some interval of frequencies. Furthermore, since the plant is open loop stable, the Nyquist plot of the return ratio $T_{N S}(s)=C_{N S} \cdot P(s)$ must not encircle the -1 critical point. As opposed to the ASFG, however, the NS controller by itself is not required to be AS.

$C_{N S}$ is designed by carefully shaping the return ratio by direct placement of poles and zeros. A zero at 4 $\mathrm{Hz}$ compensates for the bend in the original plant. Conjugate pole pairs were placed at $6.75 \mathrm{~Hz}$ and $10 \mathrm{~Hz}$ to fix the functional bandwidth at $10 \mathrm{~Hz}$. The pole pair at $10 \mathrm{~Hz}$ and a zero pair at $15 \mathrm{~Hz}$ were lightly damped in order to provide the sharp transitions seen in the slope of the return ratio at $10 \mathrm{~Hz}$ and $15 \mathrm{~Hz}$. It is this steep slope and accompanying phase lag that allows us to attain nearly $20 \mathrm{~dB}$ more negative feedback (where $\left|S_{N S}\right|<0 \mathrm{~dB}$ ) than with the ASFG controller. A pair of zeros at $60 \mathrm{~Hz}$ and a pair of poles at $200 \mathrm{~Hz}$ flatten the response to produce a Bode step. A two octave wide lead filter centered at $100 \mathrm{~Hz}$ further flattens the response and adds phase lead. The loop is shaped such that the positive feedback never exceeds $6 \mathrm{~dB}$. The gain, zeros, and poles of $C_{N S}(s)$ are $K=2119, s_{z}=(-25.13,-314.2,-14.14 \pm 93.18 i,-188.5 \pm 326.5 i)$ and $s_{p}=(-1257,-33.93 \pm 25.45 i,-12.57 \pm 61.56 i,-1257 \pm 2177 i)$.

The resulting 7 th order compensator provides nearly $38 \mathrm{~dB}$ of disturbance rejection below $10 \mathrm{~Hz}$. This is an order of magnitude greater than the ASFG controller. The $0 \mathrm{~dB}$ crossover frequency is also slightly higher at 44.5 Hz. As can be be seen in Figure 6(b), the return ratio satisfies the Nyquist stability criterion and the system without saturation is stable in closed loop. However, no physically realizable system behaves linearly under all conditions. The saturation of large signals is inevitable considering the finite nature of real world systems.

\subsection{Effects of Saturation}

The plant possesses several saturation mechanisms: the controller analog output voltage is limited to $\pm 10 \mathrm{~V}$, the maximum power output of the Techron amplifier is only a few hundred watts, the stroke of the prismatic actuator is physically limited, and the laser beam can only be deflected a few degrees before it misses the target PSD sensor. To investigate the effects of saturation on the closed loop system without driving the hardware to its physical operating limits, an additional saturation with an adjustable threshold is added to the controller output. This system is shown in Figure 7(b). This hard saturation is described by the equation

$$
S(x)= \begin{cases}t_{\text {sat }} \cdot \operatorname{sign}(x) & :|x|>t_{\text {sat }} \\ x & : \text { otherwise }\end{cases}
$$

where $S(x)$ is the saturation link output, $x$ is its input, and $t_{\text {sat }}$ is the adjustable saturation threshold. The values of $t_{\text {sat }}$ used during closed loop experimentation are sufficiently low so that this link will saturate before any of the other sources of saturation mentioned above. 


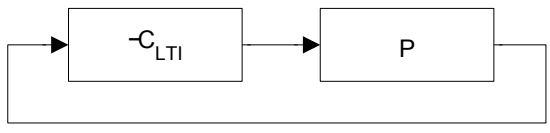

(a) Without Saturation

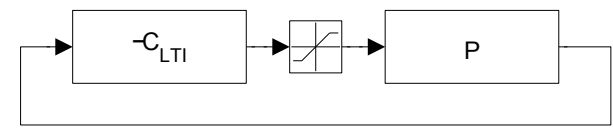

(b) With Saturation

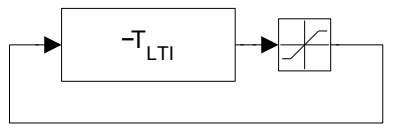

(c) Equivalent System

Figure 7. Standard Feedback Configurations and Equivalent System used for Absolute Stability Analysis

Describing functions (DF) and harmonic analysis can be useful for approximating the behavior of a nonlinear link in an linear feedback system. ${ }^{11}$ Consider a sinusoid of amplitude $E$ fed into a saturation block. For a small amplitude sinusoid, $E<t_{\text {sat }}$, the saturation link is equivalent to a unity gain. For a large amplitude sinusoid, $E>t_{\text {sat }}$, the output is a waveform of reduced amplitude but of the same frequency and phase of the input. Thus a saturation link can effectively reduce the loop gain for large signals.

Consider the Nichols plots for the ASFG and NS controllers in Figures 5(b) and 6(b). A broadband reduction of the return ratio moduli would result in the plots shifting down along the amplitude axis. Since $\left|T_{N S}\right|>0$ $\mathrm{dB}$ at some frequencies where $\arg \left(T_{N S}\right)<-180^{\circ}$, reducing the gain will eventually result in an encirclement of the critical point and the Nyquist criterion would no longer be satisfied. This is not the case for the ASFG controller.

\subsection{Absolute Stability and the Popov Criterion}

A linear time invariant (LTI) system in feedback with a nonlinearity $n(e)$ is said to be absolutely stable (AS) if it is asymptotically globally stable for any $n(e)$ satisfying the sector condition $0<e \cdot n(e)<e^{2}$. The saturation $S(x)$ in Equation 1 clearly satisfies the sector condition, so absolute stability is certainly applicable to any system equivalent to that seen in Figure 7(c).

The Popov criterion is a sufficient condition for absolute stability. ${ }^{11}$ The criterion requires that the return ratio $T(s)$ have no poles in the closed right half plane, and that there exists some $q>0$ such that $\operatorname{Re}\{(1+$ $j q \omega) \cdot T(j \omega)\}>-1$ for all $\omega$. If these conditions are met, then the closed loop system is AS. This inequality can equivalently be written as $\omega \operatorname{Im} T<q^{-1}(\operatorname{Re} T+1)$, which provides for a simple graphical interpretation. The Popov line passing through the critical point with slope $q^{-1}$ satisfies the equality $\omega \operatorname{Im} P_{L}=q^{-1}\left(\operatorname{Re} P_{L}+1\right)$. Therefore the inequality holds if the plot of $\operatorname{ReT}+j \omega \operatorname{Im} T$ on the modified Nyquist plane lies entirely to the right of some Popov line with positive slope.

All of the poles of $T_{A S F G}$ have neqative real parts and Figure 8(a) clearly shows the modified Nyquist plot for $T_{A S F G}$ lying to the right of a Popov line with slope $q^{-1}=375$. Thus the Popov criterion is satisfied and the ASFG control system truly is absolutely stable. $T_{N S}$ is also open loop stable, but by the definition of Nyquist stable there exists an $w_{0}$ such that $\arg \left(T_{N S}\left(j \omega_{0}\right)\right)=-180^{\circ}$ and $\left|T_{N S}\left(j \omega_{0}\right)\right| \geq 1$. Therefore $\operatorname{Re}\left[\left(1+q j \omega_{0}\right) T_{N S}\left(j \omega_{0}\right)\right]=$ $-\left|T_{N S}\left(j \omega_{0}\right)\right| \leq-1$ and the NS control system can not satisfy the Popov criterion.

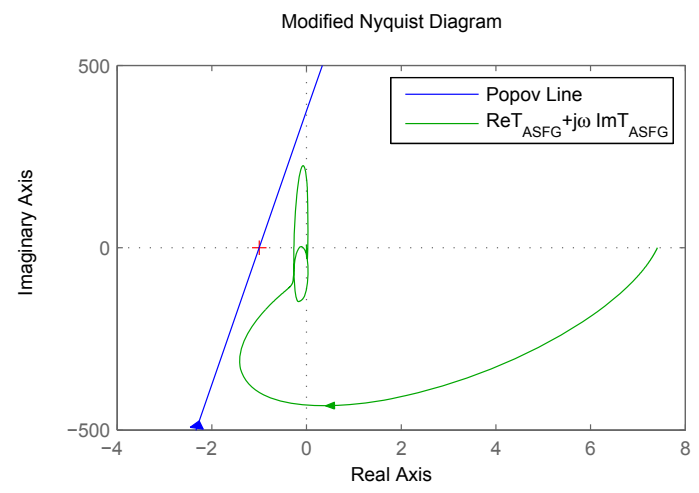

(a) ASFG Control $\left(T_{A S F G}\right)$

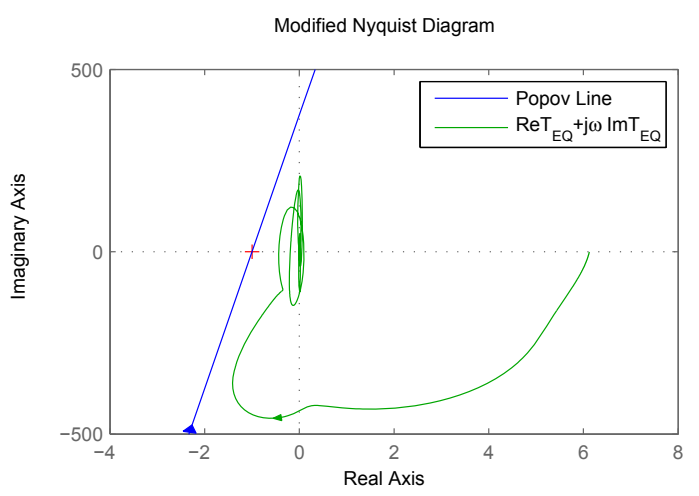

(b) NS with NDC Control $\left(T_{E Q}\right)$

Figure 8. Modified Nyquist Plots for Absolute Stability Analysis 


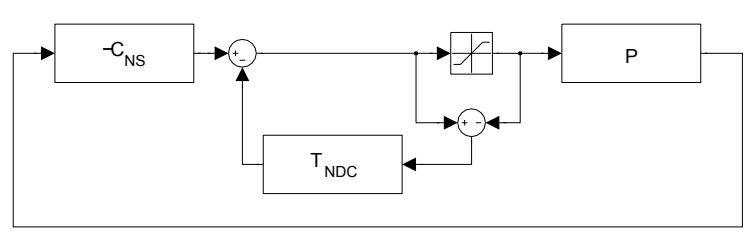

(a) NS with NDC

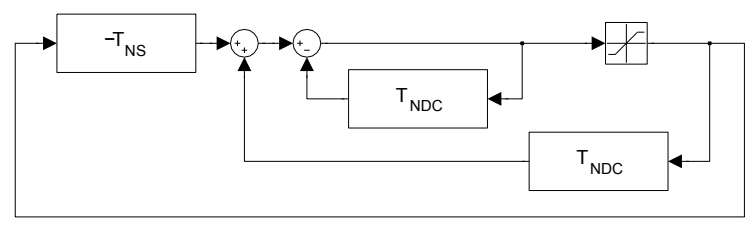

(c)

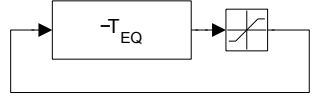

(b) Equivalent Sytem

Figure 9. Equivalent Systems for the NS with NDC Controller

\subsection{Nonlinear Dynamic Compensator (NDC) Design}

An adaptation of the control scheme in Figure 7(b) is shown in Figure 9(a). The additional feedback path is the nonlinear dynamic compensator (NDC). For small signals the output of the summing junction in the NDC path is zero. Thus, the effective return ratio for small signals is simply $T_{N S}$. For large signals, however, this difference is nonzero and the NDC feedback path is active. Therefore the NDC is able to reshape the effective return ratio of the controller for large signals.

Figures 9(a),9(c),9(d) and 9(b) illustrate a series of loop transformations used to find an equivalent system necessary for AS analysis. The return ratio of the equivalent system $T_{E Q}$ is found to be

$$
T_{E Q}=\frac{T_{N S}-T_{N D C}}{1+T_{N D C}}
$$

where $T_{N D C}$ is the return ratio of the local NDC feedback loop and $T_{N S}$ is the return ratio of the NS controller. The purpose of the NDC is to obtain an AS equivalent return ratio $T_{E Q}$. Thus $T_{A S F G}$ can serve as a target for $T_{E Q}$. For a given desired equivalent return ratio $T_{E Q}^{D}=T_{A S F G}$ and $T_{N S}$, a target NDC design $T_{N D C}^{D}$ can be found by:

$$
T_{N D C}^{D}=\frac{T_{N S}-T_{E Q}^{D}}{1+T_{E Q}^{D}}=\frac{T_{N S}-T_{A S F G}}{1+T_{A S F G}}
$$

The order of the resulting $T_{N D C}^{D}$ is quite high, and its implementation could pose serious challenges. Therefore, a lower order NDC is sought. A reduced order NDC is thus developed by the manual placement of poles and zeros. An iterative approach is indicated here as the resulting equivalent return ratio $T_{E Q}$ will differ from $T_{E Q}^{D}$, and AS will have to be verified. The resulting 9 th order $T_{N D C}$ and 40th order $T_{N D C}^{D}$ are plotted in Figure 10(a).

The gain, zeros, and poles of $T_{N D C}$ are $K=1.66 \cdot 10^{12}, s_{z}=(-1777,-46.37 \pm 61.83 i,-37.7 \pm 119.9 i)$ and $s_{p}=(-222.1,-33.93 \pm 25.45 i,-15.71 \pm 60.84 i,-942.5 \pm 1257 i,-377 \pm 2485 i)$. Well damped pole pairs at $6.75 \mathrm{~Hz}$ and $250 \mathrm{~Hz}$ in combination with lightly damped poles at $10 \mathrm{~Hz}$ and $400 \mathrm{~Hz}$ capture the sharp bends in $T_{N D C}^{D}$ seen around these frequencies. Likewise, well damped zeros at $12.3 \mathrm{~Hz}$ and lightly damped zeros at 20 $\mathrm{Hz}$ produce the remaining corner. A pole around $35 \mathrm{~Hz}$ together with a zero around $282 \mathrm{~Hz}$ helped match the modulus slope between these frequencies.

The equivalent return ratio $T_{E Q}$ of the reduced order $T_{N D C}$ and $T_{N S}$ is calculated using Equation 2, and plotted together with $T_{E Q}^{D}$ in Figure 10(b). The modified Nyquist plot of $T_{E Q}$ is shown in Figure 8(b) and lies entirely to the right of the Popov line with slope $q^{-1}=375$. $T_{E Q}$ is Hurwitz, therefore the combined NS with NDC controller is AS. 


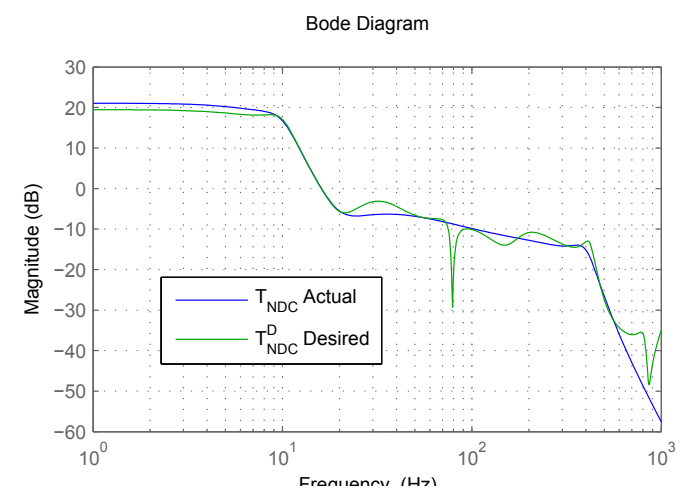

(a) $T_{N D C}$ and $T_{N D C}^{D}$

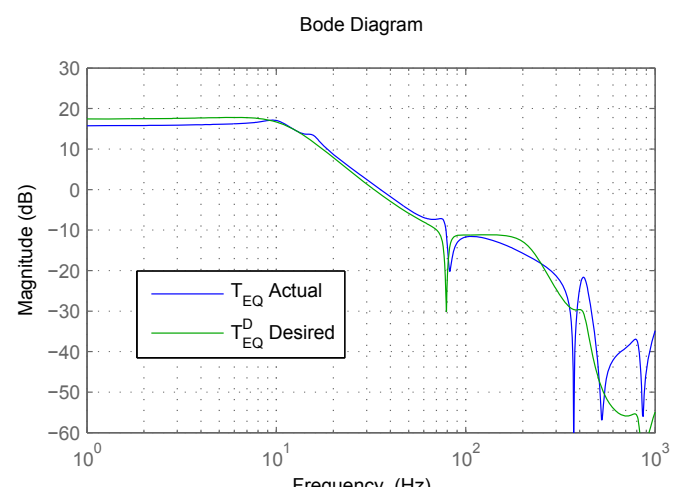

(b) $T_{E Q}$ and $T_{E Q}^{D}$

Figure 10. NDC Design

\section{CLOSED LOOP EXPERIMENTS}

\subsection{Controller Implementation}

All control design work is performed using MATLAB and the Control Systems Toolbox. The continuous time PZK models of the final control designs are discretized using Tustin's method. This bilinear transformation uses the approximation

$$
z=e^{s T}=\frac{e^{s T / 2}}{e^{-s T}} \approx \frac{1+s T / 2}{1-s T / 2}
$$

to map poles and zeros from the s-plane into the z-plane, where $T$ is the sampling time. The poles and zeros were grouped to form a cascade of second order systems. This approach of PZK design and CSOS implementation lessens the effects of finite precision arithmetic and round off errors.

Custom software developed with National Instruments LabWindows/CVI executes on a National Instruments PXI 8106 Real Time Controller. The program implements the 7 th order $C_{A S F G}, 7$ th order $C_{N S}$, and 9th order $T_{N D C}$ at a loop rate of $10 \mathrm{kHz}$. It should be noted the digitizing, processing, and outputting of signals must take no longer than $1 / 10 \mathrm{kHz}=0.1 \mathrm{~ms}$. This worst case time delay is included in the controller models during the design phase. However, a $0.1 \mathrm{~ms}$ time delay only contributes $3.6^{\circ}$ of nonminimum phase at $100 \mathrm{~Hz}$ and $36^{\circ}$ at $1 \mathrm{kHz}$. Thus it is not a major limiting factor in maximizing the crossover frequency. Companion software running on a Windows XP machine allows for loops to be open and closed, disturbances enabled or disabled, and saturation thresholds and proportional gains to be adjusted in real time.

\subsection{Disturbance Environment}

While it would be desirable to mount the mechanism on a shake table, the facility does not currently have this hardware. The robot is rigidly mounted to an air suspended optics table, so the prismatic actuator itself is used for disturbance injection. A disturbance signal is simply added to the output of the controller before it is fed to the Techron amplifier input. A plot of the disturbance generating signal is seen in Figure 11(a) along with its power spectral density (PSD) in Figure 11(b). The signal is generated by passing white noise through a $10 \mathrm{~Hz}$ first-order, low-pass filter and summing it with a $5 \mathrm{~Hz}$ sinusoidal tone.

\subsection{Closed Loop Performance}

The performance of both the ASFG and the NS with NDC controllers are verified in closed loop tests. The disturbance source described above is used in all cases, but $t_{s a t}$ is varied. A threshold of $150 \mathrm{mV}$ is sufficiently high to ensure that control effort saturation seldom occurs. In this small signal regime, the NDC is inactive and the effective return ratio of the NS with NDC controller is $T_{N S}$. This is evidenced by nearly $40 \mathrm{~dB}$ of disturbance rejection below $10 \mathrm{~Hz}$ as seen in the power spectral density plot in Figure 12(a). A saturation threshold of $50 \mathrm{mV}$ is sufficiently low to saturate the control effort some of the time. In this large signal scenario, the NDC link is active and reduces the effective return ratio to a less aggressive loop shape as shown in Figure 12(b). Comparing 


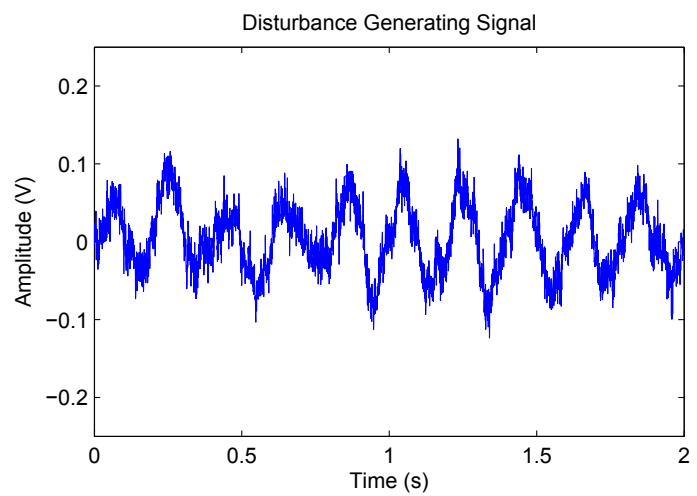

(a) Disturbance Generating Signal

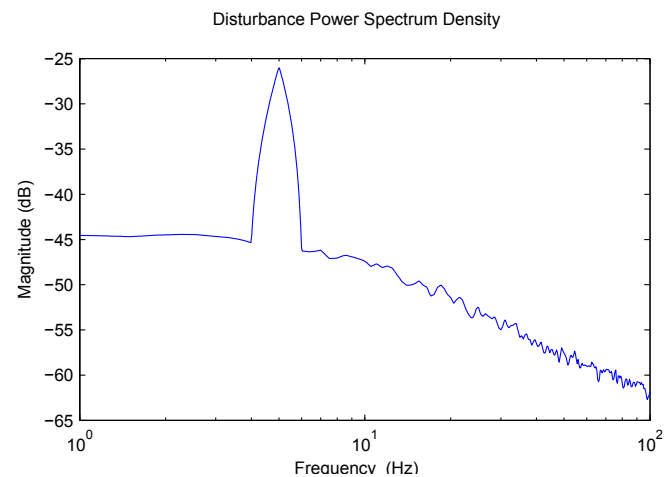

(b) Disturbance Generating PSD

Figure 11. Disturbance Generating Signal Used In Closed Loop Tests

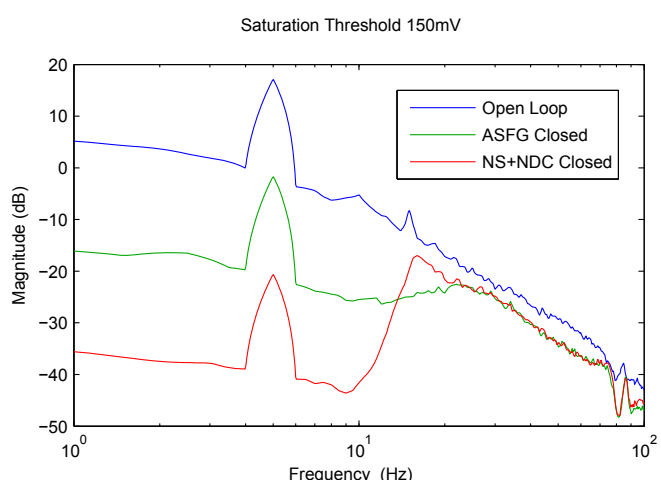

(a) Small Signal $t_{\text {sat }}=150 \mathrm{mV}$

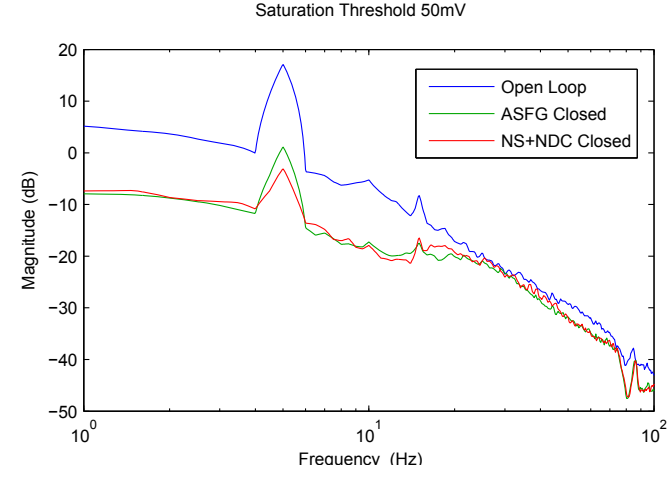

(b) Large Signal $t_{\text {sat }}=50 \mathrm{mV}$

Figure 12. Open vs Closed Loop Power Spectrum Densities

the performance of the ASFG controller between the small and large signal cases, we see that its effectiveness was also reduced in the saturated case.

The first half of Figure 13 demonstrates the unstable nature of the NS controller without the NDC. With $t_{\text {sat }}=100 \mathrm{mV}$, the NS controller without NDC is able to operate stably for some time until the control effort saturates. After this initial saturation, the control output oscillates wildly, ensuring further saturation. Stability can be regained by closing the NDC feedback path as shown in Figure 13 around the $1 \mathrm{~s}$ mark.

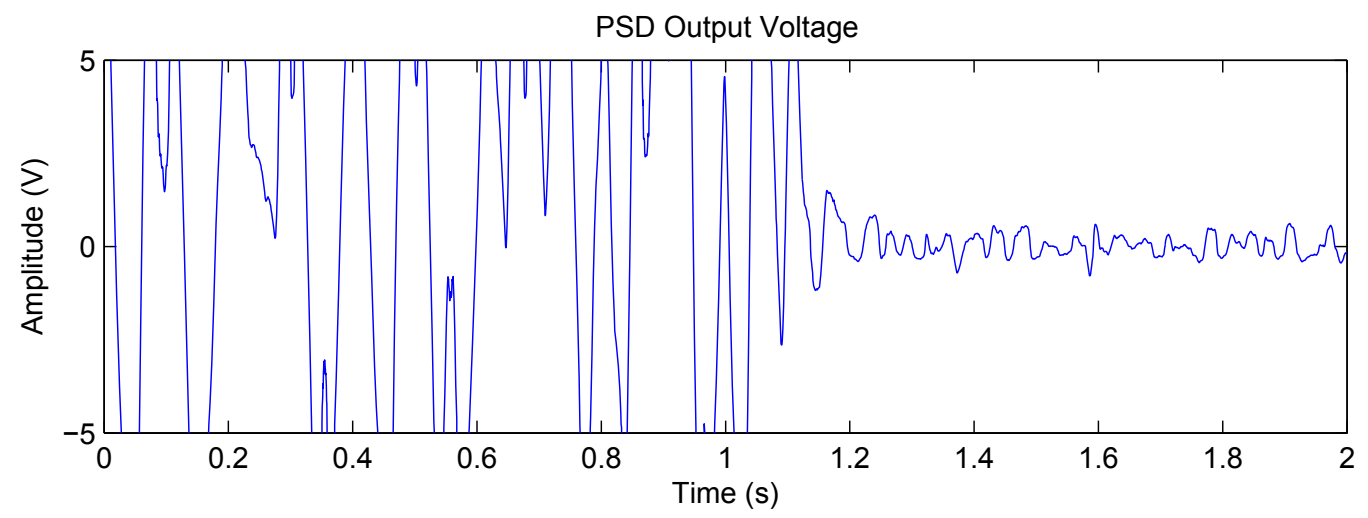

Figure 13. PSD Sensor Output Showing NS Controller Regaining Stability After NDC Loop is Enabled At Around $1 \mathrm{~s}$ 


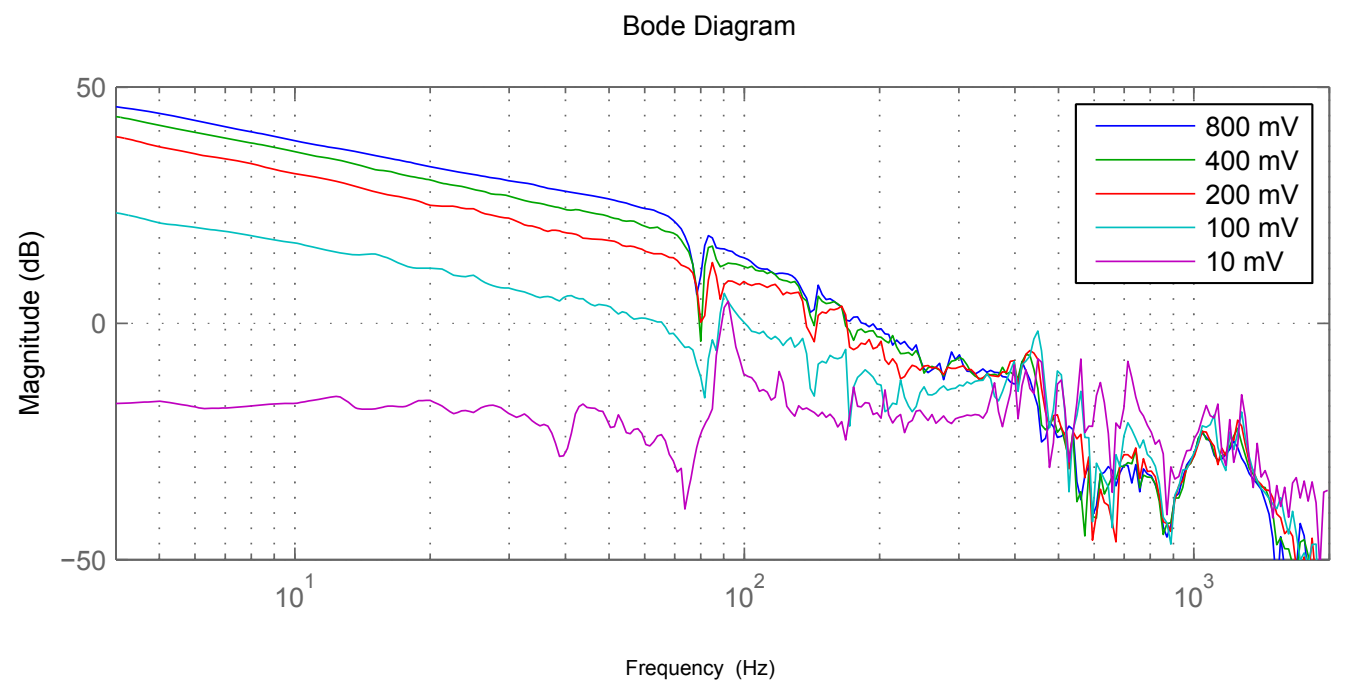

Figure 14. Plant Response to White Noise Stimulus of Differing Amplitude

\section{CONCLUSION}

Two different control strategies are applied to the prismatic axis of a limited DOF PKM, and their performance in closed loop is demonstrated. The goal is to maximize the amount of negative feedback for disturbance rejection in the frequency interval from $0 \mathrm{~Hz}$ to $10 \mathrm{~Hz}$. The linear controller $C_{A S F G}$ is shown to be absolutely stable and provides nearly $18 \mathrm{~dB}$ of feedback over the frequencies of interest. The high performance nonlinear controller consisting of a Nyquist stable controller $C_{N S}$ with nonlinear dynamic compensation $T_{N D C}$ is also shown to be absolutely stable. In the small signal case the NDC with NS is able to provide $38 \mathrm{~dB}$ disturbance rejection below $10 \mathrm{~Hz}$. This is an order of magnitude better than the ASFG controller. In the large signal case, i.e. when the control signal saturates, the nonlinear controller is able to perform at least as well as the fixed gain controller.

\section{FUTURE WORK}

Figure 14 shows the plant response to white noise stimulus of differing amplitudes. It is thought that the reduction of the plant gain for lower amplitude stimuli is due to friction. This effective drop in loop gain is similar to that caused by saturation and can cause problems for the NS controller in quiet environments. The drop in gain results in reduced stability margins and increases positive feedback. Thus, noise/disturbances around the new crossover frequency are amplified and counter the gain reducing effects of friction. An equilibrium is eventually reached as the effective return ratio becomes marginally stable and self-oscillation occurs. This phenomenon will be investigated in future work, and new control approaches will be explored.

\section{Acknowledgments}

The authors would like to thank James Nelson and Ryan Buffington for their assistance in the laboratory. This work is supported by the United States Air Force Research Laboratory and the State of Wyoming.

\section{REFERENCES}

[1] Stolle, S. and Rysdyk, R., "Flight path following guidance for unmanned air vehicles with pan-tilt camera for target observation," 22nd Digital Avionics Systems Conference, Sensing and Control (2003).

[2] Kennedy, P. and Kennedy, R., "Direct versus indirect line of sight stabilization," IEEE Transactions on Control System Technology 11(1) (2003).

[3] Biamino, D., C. G. M. M. P. A., "MAC-EYE: a tendon driven fully embedded robot eye," IEEE-RAS International Conference on Humanoid Robots 5(5) (2005).

[4] Gosselin, C.M., S. P. E. G. M., "On the development of the Agile Eye," 
[5] Bonev, I.A., C. D. and Wenger, P., "Working and assembly modes of the Agile Eye," Proceedings of the IEEE Conference on Robotics and Automation (2006).

[6] Rodriguez-Leal, E., D. J. P. G., "Inverse kinematics and motion simulation of a 2-dof parallel manipulator with 3 - $\underline{P} U P$ legs," In Computational Kinematics (2009).

[7] Vertechy, R. and Parenti-Castelli, V., "Static and stiffness analyses of a class of over-constrained parallel manipulators with legs of type US and U $\underline{P S}, "$ In IEEE Int. Conf. on Robotics and Automation (2007).

[8] Nelson, P. J., Carruthers, D. J., O’Brien, J. F., and McInroy, J. E., "Design and control of a two-arm parallel mechanism using revolute and prismatic actuators," IASTED Technology Conferences 705 (2010).

[9] Kock, S., S. W., "A parallel x-y mechanism with actuation redundancy for high-speed and active-stiffness applications," IEEE International Conference on Robotics and Automation (1998).

[10] Carruthers, D. J., O'Brien, J. F., McInroy, J. E., and Yang, Y., "Development of a voice coil-actuated limited-degree-of-freedom parallel mechanism for vibration suppression," Proceedings of the Institution of Mechanical Engineers, Part I: Journal of Systems and Control Engineering 223(1), 85-94 (2009). [DOI: 10.1243/09596518JSCE599].

[11] Lurie, B. J. and Enright, P. J., [Classical Feedback Control with MATLAB], Marcel Dekker, New York, NY (2000).

[12] O'Brien, J. F., "High order rudder roll stabilization controller with nonlinear compensation," Proceedings of the ASNE automation and control symposium (2007).

[13] O'Brien, J. F., "Rudder roll stabilization by nonlinear dynamic compensation," (2010). US Patent: US 2010/0147204 A1. 